\title{
Assignment of an impact factor to our journal
}

\author{
Yasutomo Fujii
}

(C) The Japan Society of Ultrasonics in Medicine 2010

This year an impact factor has been assigned to the official English journal of the Japan Society of Ultrasonics in Medicine. We are delighted that our journal can be internationally recognized as important one in this academic field. We would like to thank you all for your support.

In a given year, the impact factor of a journal is the average number of citations of the papers published in the journal during the two preceding years. In our case, the 2010 impact factor of our journal would be calculated as follows:

\section{0 impact factor $=A / B$}

$A=$ the number of times articles published in 2008 and 2009 were cited by indexed journals during 2010

$B=$ the total number of "citable items" published in 2008 and 2009

The impact factor is often misused to evaluate the importance of an individual publication or to assess an individual researcher. This does not work well, because a small number of publications are cited much more than the majority, and thus the importance of any one publication will be different from, and in most cases less than, the overall number.

How should we use an impact factor? The impact factor is used to compare different journals within a certain field.
Because the impact factor of a journal may reflect the number of readers, researchers will submit their work to journals that have a larger impact factor. Thus, a higher impact factor brings more submissions, leading to improvement in the quality of published scientific work. A larger number of higher-quality submissions will reduce the workload of referees. This is an excellent "cycle" not only for a journal but also for readers, including researchers.

Our journal has not yet adopted editorial policies designed to increase its impact factor. Some strategies may be necessary to achieve such a goal. These strategies may not solely involve improving the quality of published scientific work. Because review articles, which generally are cited more than research reports, can raise the impact factor of the journal, a larger percentage of review articles will be published. Several methods, not necessarily with nefarious intent, enable a journal to cite articles in the same journal, which will increase the journal's impact factor.

Finally, the editorial board welcomes more submissions of your work for publication, and hopes that a larger impact factor will be assigned to our journal and be cited on MEDLINE in the near future, which will help not only our journal but also researchers in the field of ultrasound throughout the world.
Y. Fujii ( $\square)$

Department of Clinical Laboratory Medicine,

Jichi Medical University, 3311-1 Yakushiji, Shimotsuke,

Tochigi 329-0498, Japan

e-mail: yfujii@jichi.ac.jp 